\title{
A hidden catalysis: metal-, and organocatalyst-free one-pot assembly of chiral aza- tricyclic molecules containing six contiguous stereocenters
}

\author{
Dung Tien Do ${ }^{\mathrm{a}}$ \\ ${ }^{a}$ Faculty of Chemistry, University of Science, Vietnam National University-Hanoi, 19 Le Thanh Tong, Hoan Kiem, Ha \\ Noi, Vietnam
}

E-mail: $\underline{\text { dtdung82@hus.edu.vn }}$

\begin{abstract}
Chiral molecules with their defined 3-D structures are of paramount importance for the study of chemical biology and drug discovery. Having rich structural diversity and unique stereoisomerism, chiral molecules offer a large chemical space that can be explored for the design of new therapeutic agents. ${ }^{1}$ In practice, chiral architectures are usually prepared from organometallic and organocatalytic processes where a transition metal or an organocatalyst is tailor-made for a desired reaction. As a result, developing a method that enables rapid assembly of chiral complex molecules under a metal- and organocatalyst-free condition represents a daunting challenge. Here we developed a straightforward one-pot procedure to create a chiral 3-D structure from 2-D structures and an amino acid without any chiral catalyst. The center of this research is the design of a special chiral spiroimidazolidinone cyclohexadienone intermediate, a merger of a chiral reactive substrate with multiple nucleophillic/electrophillic sites and a transient organocatalyst. This unique substrate-catalyst ("sub-catalyst") dual role of the intermediate was displayed in its aza-Michael/Michael cascade reaction with an $\alpha, \beta$-unsaturated aldehyde under an iminium/enamine catalysis. The enhanced co-ordinational proximity of the chiral substrate and catalyst in the transition state resulted in a substantial steric discrimination and an excellent overall diastereoselectivity. Aza-tricylic molecules with six contiguous stereocenters were assembled from $N$-alkylated aminophenols, $\alpha, \beta$-unsaturated aldehydes and chiral $\alpha$-amino acids under a hidden "sub-catalysis" where the strategically produced "sub-catalyst" does not present in initial components of the reaction. The success of this methodology will pave the way for many efficient preparations of chiral complex molecules.
\end{abstract}

Rapid assembly of molecules at various level of complexity is a magic power of Nature in producing diverse structures of natural products. Molecular complexity is precisely achieved from simple building blocks under biosynthetic pathways. Drawing 
inspiration from those elegant syntheses, chemists have developed numerous efficient synthetic methodologies to imitate Nature's way of producing complex molecules. ${ }^{2,3}$ Empowered by the intertwined development of organometallic, organocatalysis and enzymatic catalysis, the current state-of-the-art asymmetric synthesis could enable the preparation of most desired chiral molecules. ${ }^{4,5}$ Whereas enzymatic catalysis sets a high standard for the stereoselectivity, organometallic- and organo-catalysis have an advantage for a more diverse scope of reactions. Due to its versatile reactivity, diverse bond formation, and efficiency in chemo- and stereo-selection, organometallic catalysis has always been the preeminent tools to produce chiral molecules. ${ }^{6-11}$ In the last two decades, organocatalysis has emerged as another major tool for enantioselective synthesis. ${ }^{12}$ Ever since, domino organocatalysis has been recognized as one of the most efficient strategies to create molecules with multiple stereocenters. ${ }^{13,14}$ Among a plethora of available methods for rapidly building up molecular complexity, oxidative dearomatization is a powerful technique to convert 2-D flat structures into 3-D organic structures in numerous domino organocatalytic processes. ${ }^{15-19}$ Another substantial strategy to create stereochemically rich architectures is operating local desymmetrization under organocatalytic conditions. Engineering an enantiotopic group discrimination, local desymmetrization rapidly transforms prochiral or meso-molecules into chiral complex molecules with high conformity. ${ }^{20,21}$ Generally, a chiral organometallic or an organocatalyst is an essential part of most reactions designed to create a complex architecture. Therefore, rapid assembly of chiral molecules with multiple stereocenters from simple building blocks under a one-pot metal- and organocatalyst-free condition is a daunting challenge. To accomplish this task, it is crucial that individual components would be arranged to react and produce chiral advanced intermediates that could engineer the next highly diastereoselective transformations. Among early development of metal-free synthesis of chiral molecules, MacMillan's pioneer works on iminium/enamine catalysis of chiral imidazolidinones have inspired ample applications of these molecules in numerous asymmetric transformations. ${ }^{22-25}$ However, the lack of viable functionalities within their structures for extended elaboration has limited a broader synthetic application of the imidazolidinones. Designing a new class of chiral imidazolidinones that possess viable functional groups embedded within their structures would present a substantial synthetic opportunity. Allured by a functionality-rich chiral species that could also play a role of a versatile organocatalyst in chemical reactions, we identified chiral spiroimidazolidinone cyclohexadienone (scheme 1) as a special intermediate for our quest to develop a method that can quickly buildup molecular complexity. Structurally, spiroimidazolidinone cyclohexadienone possesses a chiral imidazolidinone template with an aza-nucleophillic site and a 1,4-cyclohexadienone core. With its unique symmetrical cyclic structure and rich functionalities, the cyclohexadienone is an attractive template to build complex cyclic architectures. It is beneficial that the substructure can be readily prepared from an oxidative dearomatization reaction of a phenol derivative. Numerous transition metal catalysis and organocatalysis have been utilized for the asymmetric desymmetrization of cyclohexadienone substrates. ${ }^{26-}$ 42 Surprisingly, aza-Michael/Michael cyclization, a potentially powerful reaction to construct aza-cyclic complex molecules, has only been applied for the asymmetric desymmetrization of para-quinamines using prolinol silyl ethers to promote iminium/enamine activation of $\alpha, \beta$-unsaturated aldehydes. ${ }^{43}$ The aspiration for our research is that the special structure of spiroimidazolidinone cyclohexadienone could unlock some potential stereoselective reactivities. We envisioned that a chiral spiroimidazolidinone cyclohexadienone could potentially undergo a sequence of aza-Michael/Michael addition with a Michael acceptor to generate a tricyclic molecule containing six contiguous stereocenters. If the cascade could be combined with a onepot synthesis of the chiral spiroimidazolidinone cyclohexadienones from amino acids and $N$-alkylated-p-aminophenols, we envisage a straightforward route to produce a tricyclic molecule, containing six contiguous stereocenters simply by mixing a chiral amino acid with a $\mathrm{N}$-alkylated-p-aminophenol and a Michael acceptor under a one-pot condition (scheme 1). Although realization of this strategy would solve an unprecedented task of creating a 3-D structure from 2-D structures without any chiral catalyst, there are several substantial challenges that need to be addressed. (a) Establish a one-pot reaction to prepare a chiral spiroimidazolidinone cyclohexadienone from an amino acid and a $N$-alkylated-p-aminophenol. This unsolved task needs to be developed from the ground up (b) Realize the aza-Michael/Michael addition cascade and derive a high diastereoselectivity for the overall transformation without an external chiral catalyst. Essentially, the chirality of an amino acid precursor would control the overall stereochemistry of the reaction. We recognized that enabling chemical reactivity of an intrinsic weak nucleophile such as an imidazolidinone and controlling the formation of six contiguous stereocenters in this single pot process are the most challenging quests. (c) Establish an overall one pot sequence while ensuring that reagents or solvents from previous steps do not interfere with the sequential reactions. It is a demanding task as many distinctive bond-forming and bond-breaking events would occur during the whole process.

We began our study of a one pot synthesis of the chiral sprioimidazolidinone cylohexadienone from a chiral amino acid and a $N$ alkylated-p-aminophenol (scheme 2). Deviating from prevalent reported syntheses of chiral imidazolidinones via the condensation of amino amides and carbonyl compounds ${ }^{44}$, we intended to develop the straightforward synthesis of spiroimidazolidinone cyclohexadienones from an amide coupling/oxidative dearomatization sequential reaction. First, the oxidative dearomatization of an $\mathrm{N}$-Boc-protected amide coupling adduct (scheme 2, reaction 1) was investigated with hypervalent iodine reagents in various organic solvents (table $1, \mathrm{SI}$ ). Whereas most solvents and hypervalent iodine reagents 
failed to deliver the desired products, we were satisfied to achieve the oxidative dearomatization using diacetoxyiodo benzene (DIB) in 2,2,2-trifluoroethanol (TFE) (For details see Supporting information). An addition of urea to the reaction did not affect the outcome of the cascade and the combination of TFE with solvent such as acetonitrile or dichloromethane (DCM) also produced the desired product with satisfied yields. Unfortunately, attempts to achieve a one-pot procedure of amide coupling/oxidative dearomatization in TFE was meet with no success. The one-pot procedure was realized when DCM was used as the sole solvent in the amide coupling step before TFE was added to the reaction (DCM/TFE, 1:2) to furnish the oxidative dearomatization. The imidazolidinone lla was isolated with $65 \%$ yield (Scheme 2, reaction 2) and its structure was unambiguously assigned from the X-ray crystallography. The free amine Illa was then produced with good yield (60\% yield, scheme 2, reaction 3) under a one-pot amide coupling/oxidative dearomatization/deprotection procedure.

This research aims to create six contiguous stereocenters around a tricyclic molecule with a high stereoselectivity. Since the chirality of the amino acid is embedded into the spiroimidazolidinone, the overall stereoselectivity of the process would depend on the stereoselectivity of the aza-Michael/Michael addition sequence. However, the steric discrimination for facial approach of the spiroimidazolidinone cyclohexadienone to a substituted $C=C$ double bond is apparently small (scheme 3 , box 1 ). Therefore, the stereoselectivity of an aza-Michael addition step would be predictably undesirable. Another challenging aspect of realizing the desired aza-Michael/Michael addition is that an imidazolidinone is typically an intrinsic weak nucleophile. ${ }^{45}$ Indeed, in a quick survey of the cyclization of the amine Illa with Michael acceptors such as nitro styrene, E-chalcone and Ethyl $\beta$-( $p$-MeOPhenyl)acrylate, no reaction was observed under these conditions (table 2, SI).

We envisioned that the lackluster reactivity of an imidazolidinone and the challenging face-selection of the azaMichael/Michael cascade could be addressed if we use an $\alpha, \beta$-unsaturated aldehyde for this reaction. The chiral substrate imidazolidinone could also be an iminium/enamine catalyst to activate the $\alpha, \beta$-unsaturated aldehyde, promoting a selfcatalyzed aza-Michael/Michael cyclization (scheme 3, box 2). Under this iminium/enamine catalysis, amplified electrophilicity of the iminium would accommodate for the low nucleophilicity of the imidazolidinone. This substrate-catalyst (sub-catalyst) dual role of the imidazolidinone would enhance a co-ordinational proximity of the chiral substrate and the catalyst then magnify the steric discrimination of facial attacks of the aza-nucleophile onto the iminium in the transition state. A Re-face or Si-face attack of the aza-nucleophile would prevail depending on the absolute stereochemistry of the chiral imidazolidinone. Specifically, for a (S)-imidazolidinone, the R' group from the catalyst would effectively obstruct the Re-face of the iminium and therefore favor a Si-face attack (scheme 3, box 2). Similarly, an (R)-imidazolidinone would favor a Re-face attack. This face-discrimination approach of the aza-nucleophile would create a new C-N bond and set the stereochemistry at the $\beta$ carbon, originally from the $\alpha, \beta$-unsaturated aldehyde. The resulting enamine would then undergo a cascade intramolecular Michael addition to one of the two diastereotopic vinylic ketones either from Si-face (path a, red arrow) or Re-face (path b, green arrow) under enamine catalysis (scheme 3, box 3). With an (S)-imidazolidinone, in one hand, the Re-face attack would be highly disfavored as it suffers from two significant steric interactions. The first one is the developing steric interaction of $R^{\prime}$ from in the substrate with the ring-forming pyrrolidine. The second one is the interaction of the catalyst site with the cyclohexenone core of the substrate. On the other hand, the Si-face attack would avoid these steric cumbers. The formation of the new $\mathrm{C}$ - $\mathrm{C}$ bond from this diastereotopic selection (path a over path b) would then lock the nitrogen in a rigid aza-tricyclic framework and concurrently operate a desymmetrization of the cyclohexadienone ring. Essentially, this final Michael addition step would define the simultaneous formation of the four new stereogenic centers including stereochemically challenging features such as a spiroaminal and a nitrogenic stereocenter. Overall, this feature would induce a desired reactivity and stereoselectivity for the construction of an aza-tricyclic system which contains six contiguous stereocenters.

The next task is to realize a suitable condition for the aza-Michael/Michael cascade of the spiroimidazolidinone and an $\alpha, \beta$ unsaturated aldehyde. When a mixture of the amine (IIla) and (E)-cinnamaldehyde (C1) in DCM for 24 hours showed no reaction, several Bronsted acids were probed to promote the aza-Michael/Michael cascade (Table 3, SI). ${ }^{46}$ Complex mixture with no desired product were observed in most cases. Only acetic acid (AcOH) and TFA delivered the desired product with excellent diastereoselectivity (>20:1 dr). TFA proved to be superior as it produced an excellent outcome $(90 \%,>20: 1 \mathrm{dr}$ ). Interestingly, when the reaction was run in mixture of DCM/TFE (1:2) solutions, no desired product was isolated. This result suggests that TFE is not suitable for this aza-Michael/Michael cascade reaction. With every single step was realized, it is still challenging to establish a combined one-pot condition for overall process. When triflic acid (2 equivalents) or excess (10 equivalents) of TFA, typical for Boc-deprotections, was added with E-cinnamaldehyde $\mathbf{C} 1$ to a one-pot amide coupling/oxidative dearomatization of N-Boc-L-Phenylalanine (A1), aminophenol (B1), no desired product D1 was isolated under the one-pot sequence (see SI for details). Quenching excess of these acids from the Boc-deprotection step with weak bases such as triethyl amine or solid sodium bicarbonate only resulted in forming complex mixtures. Apparently, the desired one-pot condition in the aza-Michael/Michael cascade has to alleviate the undesired role of the excessive acids (TFA or triflic acid) and unsuitable 
solvent (TFE). We noticed that, unlike most of metal-catalyzed reactions, water can sometimes be added to an organocatalyzed reaction. ${ }^{47}$ We envisioned that, adding water to the reaction would create a two-phase solution where aqueous phase would extract excess acids and TFE, while the organic phase (DCM) contains protonated amine with the aldehyde. This two-phase condition would be suitable for the aza-Michael/Michael cyclization. Delightfully, a one-pot condition using 1.5 equivalent of $N$ Boc-L-phenylalanine (A1), 1.0 equivvalent of aminophenol (B1) and 2.0 equivalent of E-cinnamaldehyde (C1) produced cyclization adduct D1 with moderate yield (55\%) and excellent diastereoselectivity (>20:1), (scheme 2, reaction 5). Using this one-pot procedure, the scope of $\mathrm{N}$-substituted aminophenols was first explored (D1-10, scheme 4). Substrates with electrondonating or electron-withdrawing groups produced desired products with satisfied yields with the reaction was tolerated with halogens at $o_{-}, m-$, and $p$-position in the benzene ring of the amine. The substituents in the phenyl ring of the substrates generally do not exhibit a significant effect on the reaction as we observed a slightly drop in the reaction yield of ortho-methyl substitution (D2, scheme 4). Next, the scope of $\alpha, \beta$-unsaturated aldehydes was examined and the reaction delivered the desired products with consistent moderate yields and >20:1 dr (D11-16, scheme 4). Other protected $\alpha$-amino acids were then investigated (D17-23, scheme 4). N-Boc- $\alpha$-amino acids such as N-Boc-L-valine (D18), O-Benzyl-N-Boc-L-tyrosine (D19), N-Boc-Lisoleucine (D21), O-benzyl-N-Boc-L-threonine (D22) consistently produced the desired products with moderate to good yields and >20:1 dr. For substrates such as N-Boc-L-alanine (D17) and N-Boc-L-leucine (D20), we found that a two-step procedure was necessary to achieve the desired products with excellent diastereoselectivity as the one-pot generated a complex mixture. In fact, we need to quench the reaction after the Boc-deproctection and removed all the solvents before the aza-Michael/Michael addition was proceed in DCM in the presence of TFA (SI). Interestingly, when we employed N-Boc-D-phenylalanine in place of $\mathrm{N}$-Boc-L-phenylalanine under the one pot condition with $\mathbf{C} 1$ and B1, the reaction produced D23, the enantiomer of D1, with an equal efficiency (55\% yield, >20:1 dr). When we used (Z)-cinnamaldehyde in either the one-pot procedure or the TFE-catalyzed aza-Michael/Michael addition with the free amine Illa with an expectation that a diastereomer of D1 would be produced. However, no other diastereomers was observed in the reaction and D1 was always the only cyclization adduct (SI). We noted that, under the reaction condition, the excess cinnamaldehyde was completely converted to E-isomer. This result demonstrates a fast $\mathrm{E} / \mathrm{Z}$ isomerization of cinnamaldehyde under a quick reversible aza-Michael/retro aza-Michael process.

In general, the representative scope showcased the structural flexibility of the precursors and demonstrated that this methodology can deliver a large library of derivatives. To further highlight the potential applicability of this methodology, a one-pot reaction was carried out at $10 \mathrm{mmol}$ scale. When A1, B1 and C1 were subjected to the one-pot procedure with technical grade reagents under ambient atmosphere, $2.62 \mathrm{~g}$ of the desired product D1 was isolated with $55 \%$ yield and excellent $d r(>20: 1 d r)$ (scheme 5).

\section{Mechanism}

While the proposed iminium catalysis helps explain for the excellent diastereoselectivity for the aza-Michael addition step, this feature, regarding reaction profile, could also be accounted for by a Curtin-Hammett kinetics mechanism where the azaMichael addition is reversible and unselective, then only one of the diastereomers cyclizes irreversibly to the final product. To probe into the proposed iminium/enamine catalysis, a ${ }^{1} \mathrm{H}-\mathrm{NMR}$ profile of reaction mixture at various ratio of IIIa (1.0; $1.0 ; 1.5$ equiv), E-cinnamaldehyde (15.0; 1.0; 1.0 equiv) and TFA (2.0 equiv) in $\mathrm{CDCl}_{3}$ was monitored (table 4 , SI). A direct $\mathrm{Br} \varnothing n s t e d$ acidcatalyzed mechanism is ruled out as no direct aza-Michael addition adduct was observed from reaction intermediates. The iminium/enamine formation which correlates with the significant drop in relative concentration of the free E-cinnamaldehyde in the reaction mixtures, was observed with some distinctive peaks in ${ }^{1} \mathrm{H}-\mathrm{NMR}$. Additionally, treatment of the reaction mixture with water after one-hour showcased an acid-catalyzed iminium/Michael addition/enamine formation-hydrolysis/retro azaMichael addition transformation where a complex ${ }^{1} \mathrm{H}-\mathrm{NMR}$ spectrum of reaction mixture turned into a ${ }^{1} \mathrm{H}$ NMR spectrum with distinctive pattern which includes only free amine IIla, the E-cinnamaldehyde and a small portion ( $8 \%$ conversion) of cyclization adduct D1 (see SI for more information). These experiments unambiguously evidenced an iminium/enamine catalysis.

The relative structural configuration of products from our proposed mechanism was supported by NOESY NMR of D15 (see supporting information for details). 
Apart from the tertiary stereogenic center originated from the amino acid precursor, all the five newborn stereocenters were constructed with control in tandem during the cascade of aza-Michael/Michael addition/desymmetrization. Overall, the stereoselectivity of the whole process is controlled only by the chirarity of an amino acid precursor. The strategically produced imidazolidinone intermediates showcases a hidden substrate-catalyst dual role to promote sequential self-catalyzed reactions. Under an operational simple one-pot procedure, the aza-tricyclic products were prepared with moderate to good yield and excellent diastereoselectivity. However, the efficiency of the process is inadvertently disguised as this one-pot procedure involves five bond-forming events. Given the aza-tricyclic adducts possess many distinctive functional groups for structural elaboration and most known therapeutic agents contain heteroatoms with a $\mathrm{C}-\mathrm{N}$ bond formation presents in more than $80 \%$ of the most commonly employed chemical reactions in the discovery of new medicines 48 , we foresee our methodology can provide a new approach to prepare, with high chemo-and stereoselectivity, complex molecules as potential pharmaceutical candidates.

References:

1. W. H. Brooks \& W. C. Guida and K. G. Daniel. The Significance of Chirality in Drug Design and Development. Curr. Top. Med. Chem. 11, 760-770 (2011).

2. Mohr, J. T., Krout, M. R. \& Stoltz, B. M. Natural products as inspiration for the development of asymmetric catalysis. Nature 455, 323-332 (2008).

3. Chen, K. \& Arnold, F. H. Engineering new catalytic activities in enzymes. Nat. Catal. 3, 203-213 (2020).

4. Hönig, M., Sondermann, P., Turner, N. J. \& Carreira, E. M. Enantioselective Chemo- and Biocatalysis: Partners in Retrosynthesis. Angew. Chem. Int. Ed. 56, 8942-8973 (2017).

5. Taylor, M. S. \& Jacobsen, E. N. Asymmetric catalysis in complex target synthesis. Proc. Natl. Acad.

Sci. U. S. A. 101, 5368 (2004).

6. Toste, F. D. \& You, S.-L. Asymmetric Synthesis Enabled by Organometallic Complexes.

Organometallics 38, 3899-3901 (2019).

7. Zhou, Q.-L. Transition-Metal Catalysis and Organocatalysis: Where Can Progress Be Expected?

Angew. Chem. Int. Ed. 55, 5352-5353 (2016).

8. Vargová, D., Némethová, I., Plevová, K. \& Šebesta, R. Asymmetric Transition-Metal Catalysis in the Formation and Functionalization of Metal Enolates. ACS Catal. 9, 3104-3143 (2019). 
9. Noyori, R. Asymmetric Catalysis: Science and Opportunities (Nobel Lecture). Angew. Chem. Int. Ed. 41, 2008-2022 (2002).

10. Cherney, A. H., Kadunce, N. T. \& Reisman, S. E. Enantioselective and Enantiospecific TransitionMetal-Catalyzed Cross-Coupling Reactions of Organometallic Reagents To Construct C-C Bonds. Chem. Rev. 115, 9587-9652 (2015).

11. Blaser, H.-U. Industrial asymmetric catalysis: Approaches and results. Rendiconti Lincei 18, 281-304 (2007).

12. MacMillan, D. W. C. The advent and development of organocatalysis. Nature 455, 304-308 (2008).

13. Grondal, C., Jeanty, M. \& Enders, D. Organocatalytic cascade reactions as a new tool in total synthesis. Nat. Chem. 2, 167-178 (2010).

14. Enders, D., Hüttl, M. R. M., Grondal, C. \& Raabe, G. Control of four stereocentres in a triple cascade organocatalytic reaction. Nature 441, 861-863 (2006).

15. Roche, S. P. \& Porco, J. A., Jr. Dearomatization strategies in the synthesis of complex natural products. Angew. Chem. Int. Ed Engl. 50, 4068-4093 (2011).

16. Reddy, C. R., Prajapti, S. K., Warudikar, K., Ranjan, R. \& Rao, B. B. ipso-Cyclization: an emerging tool for multifunctional spirocyclohexadienones. Org. Biomol. Chem. 15, 3130-3151 (2017).

17. Pouységu, L., Deffieux, D. \& Quideau, S. Hypervalent iodine-mediated phenol dearomatization in natural product synthesis. Tetrahedron 66, 2235-2261 (2010).

18. Vo, N. T., Pace, R. D. M., O'Har, F. \& Gaunt, M. J. An Enantioselective Organocatalytic Oxidative Dearomatization Strategy. J. Am. Chem. Soc. 130, 404-405 (2008).

19. Baker Dockrey, S. A., Lukowski, A. L., Becker, M. R. \& Narayan, A. R. H. Biocatalytic site- and enantioselective oxidative dearomatization of phenols. Nat. Chem. 10, 119-125 (2018).

20. Horwitz, M. A. \& Johnson, J. S. Local Desymmetrization through Diastereotopic Group Selection: An Enabling Strategy for Natural Product Synthesis. Eur. J. Org. Chem. 2017, 1381-1390 (2017). 
21. Borissov, A. et al. Organocatalytic enantioselective desymmetrisation. Chem. Soc. Rev. 45, 54745540 (2016).

22. Paras, N. A. \& MacMillan, D. W. C. New Strategies in Organic Catalysis: The First Enantioselective Organocatalytic Friedel-Crafts Alkylation. J. Am. Chem. Soc. 123, 4370-4371 (2001).

23. Erkkilä, A., Majander, I. \& Pihko, P. M. Iminium Catalysis. Chem. Rev. 107, 5416-5470 (2007).

24. Lelais, G. \& MacMillan, D. Modern strategies in organic catalysis: The advent and development of iminium activation. Aldrichimica Acta 39, 79-87 (2006).

25. Liu, Y.-Y., Liu, J., Lu, L.-Q. \& Xiao, W.-J. Organocatalysis Combined with Photocatalysis. Top. Curr. Chem. 377, 37 (2019).

26. Imbos, R., Minnaard, A. J. \& Feringa, B. L. A Highly Enantioselective Intramolecular Heck Reaction with a Monodentate Ligand. J. Am. Chem. Soc. 124, 184-185 (2002).

27. Hayashi, Y. et al. Cysteine-Derived Organocatalyst in a Highly Enantioselective Intramolecular Michael Reaction. J. Am. Chem. Soc. 127, 16028-16029 (2005).

28. Liu, Q. \& Rovis, T. Asymmetric Synthesis of Hydrobenzofuranones via Desymmetrization of Cyclohexadienones Using the Intramolecular Stetter Reaction. J. Am. Chem. Soc. 128, 2552-2553 (2006).

29. Rubush, D. M., Morges, M. A., Rose, B. J., Thamm, D. H. \& Rovis, T. An Asymmetric Synthesis of 1,2,4-Trioxane Anticancer Agents via Desymmetrization of Peroxyquinols through a Brønsted Acid Catalysis Cascade. J. Am. Chem. Soc. 134, 13554-13557 (2012).

30. Ratnikov, M. O., Farkas, L. E. \& Doyle, M. P. Tandem Sequence of Phenol Oxidation and Intramolecular Addition as a Method in Building Heterocycles. J. Org. Chem. 77, 10294-10303 (2012). 
31. Tello-Aburto, R., Kalstabakken, K. A., Volp, K. A. \& Harned, A. M. Regioselective and stereoselective cyclizations of cyclohexadienones tethered to active methylene groups. Org. Biomol. Chem. 9, 7849-7859 (2011).

32. Huang, X., Liu, M., Jasinski, J. P., Peng, B. \& Zhang, W. Recyclable Organocatalysts for a One-Pot Asymmetric Synthesis of 2-Fluorocyclohexanols Bearing Six Contiguous Stereocenters. Adv. Synth. Catal. 359, 1919-1926 (2017).

33. Gu, Q. \& You, S.-L. Desymmetrization of cyclohexadienones viacinchonine derived thioureacatalyzed enantioselective aza-Michael reaction and total synthesis of (-)-Mesembrine. Chem. Sci. 2, 1519-1522 (2011).

34. Breuning, M. \& Corey, E. J. Catalytic Enantioselective Diels-Alder Reactions of 1,4-Quinone Monoketals. Org. Lett. 3, 1559-1562 (2001).

35. Chauhan, P., Urbanietz, G., Raabe, G. \& Enders, D. Asymmetric synthesis of functionalized cyclohexanes bearing five stereocenters via a one-pot organocatalytic Michael-Michael-1,2addition sequence. Chem. Commun. 50, 6853-6855 (2014).

36. Corbett, M. T. \& Johnson, J. S. Enantioselective synthesis of hindered cyclic dialkyl ethers via catalytic oxa-Michael/Michael desymmetrization. Chem. Sci. 4, 2828-2832 (2013).

37. Leon, R., Jawalekar, A., Redert, T. \& Gaunt, M. J. Catalytic enantioselective assembly of complex molecules containing embedded quaternary stereogenic centres from simple anisidine derivatives. Chem. Sci. 2, 1487-1490 (2011).

38. Keilitz, J., Newman, S. G. \& Lautens, M. Enantioselective Rh-Catalyzed Domino Transformations of Alkynylcyclohexadienones with Organoboron Reagents. Org. Lett. 15, 1148-1151 (2013).

39. Jia, M.-Q. \& You, S.-L. Desymmetrization of cyclohexadienones viad-camphor-derived triazolium salt catalyzed intramolecular Stetter reaction. Chem. Commun. 48, 6363-6365 (2012). 
40. Jia, M.-Q., Liu, C. \& You, S.-L. Diastereoselective and Enantioselective Desymmetrization of $\alpha$ Substituted Cyclohexadienones via Intramolecular Stetter Reaction. J. Org. Chem. 77, 10996-11001 (2012).

41. Wu, W. et al. Asymmetric Intramolecular Oxa-Michael Reactions of Cyclohexadienones Catalyzed by a Primary Amine Salt. Angew. Chem. Int. Ed. 52, 1743-1747 (2013).

42. Takizawa, S., Nguyen, T. M.-N., Grossmann, A., Enders, D. \& Sasai, H. Enantioselective Synthesis of

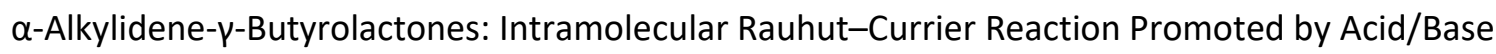
Organocatalysts. Angew. Chem. Int. Ed. 51, 5423-5426 (2012).

43. Pantaine, L., Coeffard, V., Moreau, X. \& Greck, C. Enantioselective Desymmetrization of paraQuinamines through an Aminocatalyzed Aza-Michael/Cyclization Cascade Reaction. Org. Lett. 17, 3674-3677 (2015).

44. Blackmore, T. \& Thompson, P. IMIDAZOLIDIN-4-ones: their syntheses and applications. Heterocycles 83, 1953-1975 (2011).

45. An, F., Maji, B., Min, E., Ofial, A. R. \& Mayr, H. Basicities and Nucleophilicities of Pyrrolidines and Imidazolidinones Used as Organocatalysts. J. Am. Chem. Soc. 142, 1526-1547 (2020).

46. Xu, Z., Buechler, T., Wheeler, K. \& Wang, H. A Three-Component Reaction Based on a RemoteGroup-Directed Dynamic Kinetic Aza-Michael Addition: Stereoselective Synthesis of Imidazolidin-4ones. Chem. - Eur. J. 16, 2972-2976 (2010).

47. van der Helm, M. P., Klemm, B. \& Eelkema, R. Organocatalysis in aqueous media. Nat. Rev. Chem. 3, 491-508 (2019).

48. Roughley, S. D. \& Jordan, A. M. The Medicinal Chemist's Toolbox: An Analysis of Reactions Used in the Pursuit of Drug Candidates. J. Med. Chem. 54, 3451-3479 (2011). 
Acknowledgements: The author is grateful to Mac Group at Vietnam National University and Wright lab at the University of Connecticut for sharing lab facility. The author thanks Dr Son Nguyen for his assistance on the HRMS measurement of compounds and Dr. Thang Pham for the X-ray crystallography of compound Ila. The author is grateful to Dr. Linh Nguyen for proofreading the manuscript and supporting this research.

\section{TOC}

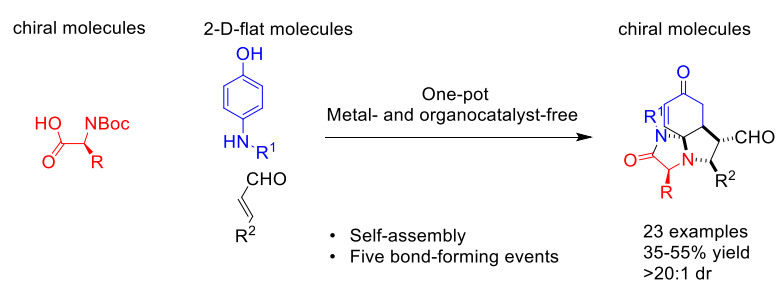

Scheme 1. Conceptual design

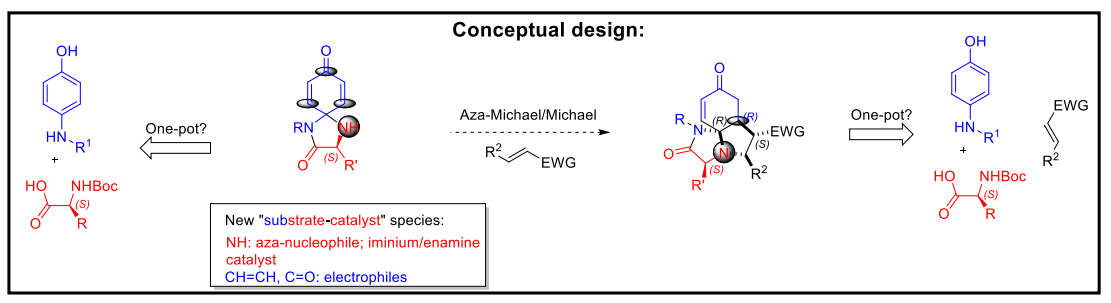

Scheme 2. Screening conditions
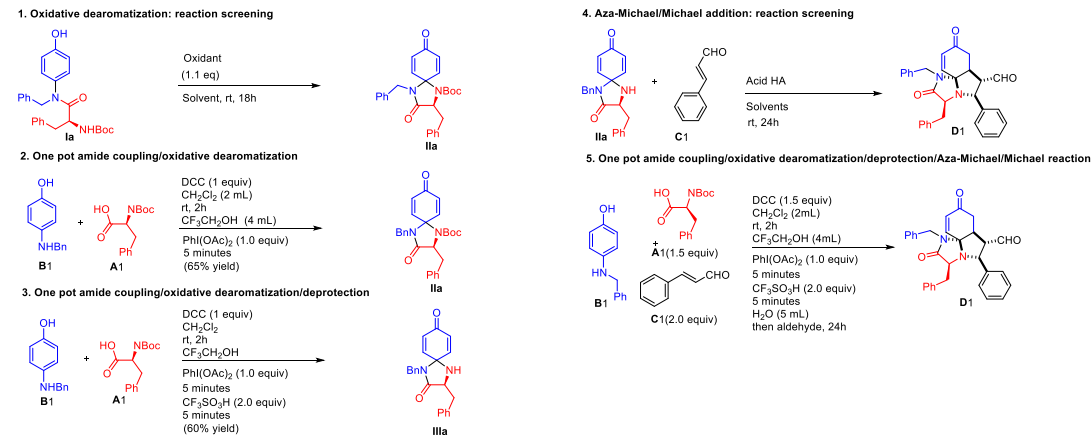
Scheme 3. Mechanistic proposal for the Aza-Michael/Michael addition/desymmetrization
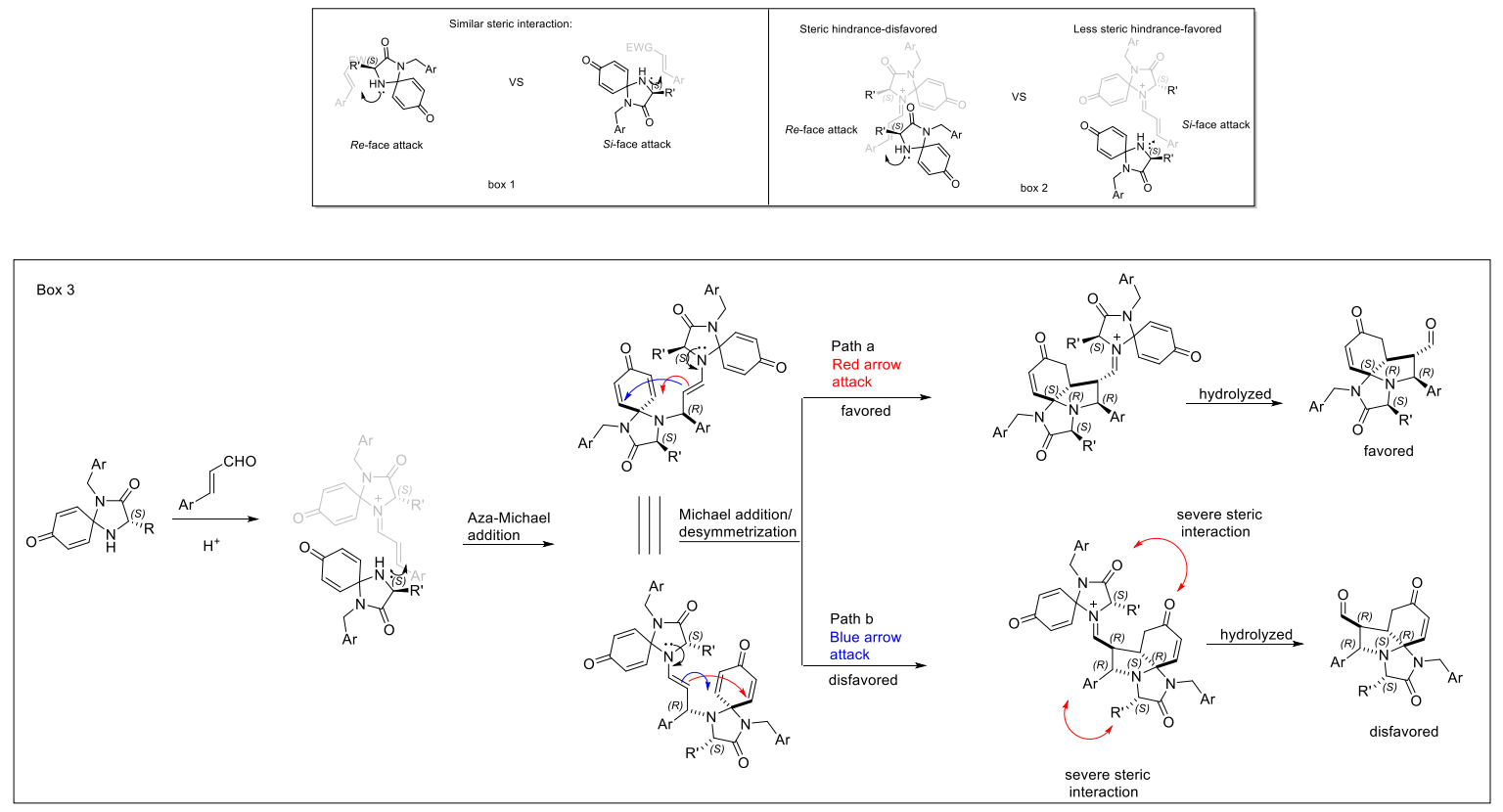

Scheme 4. Scope of the reaction

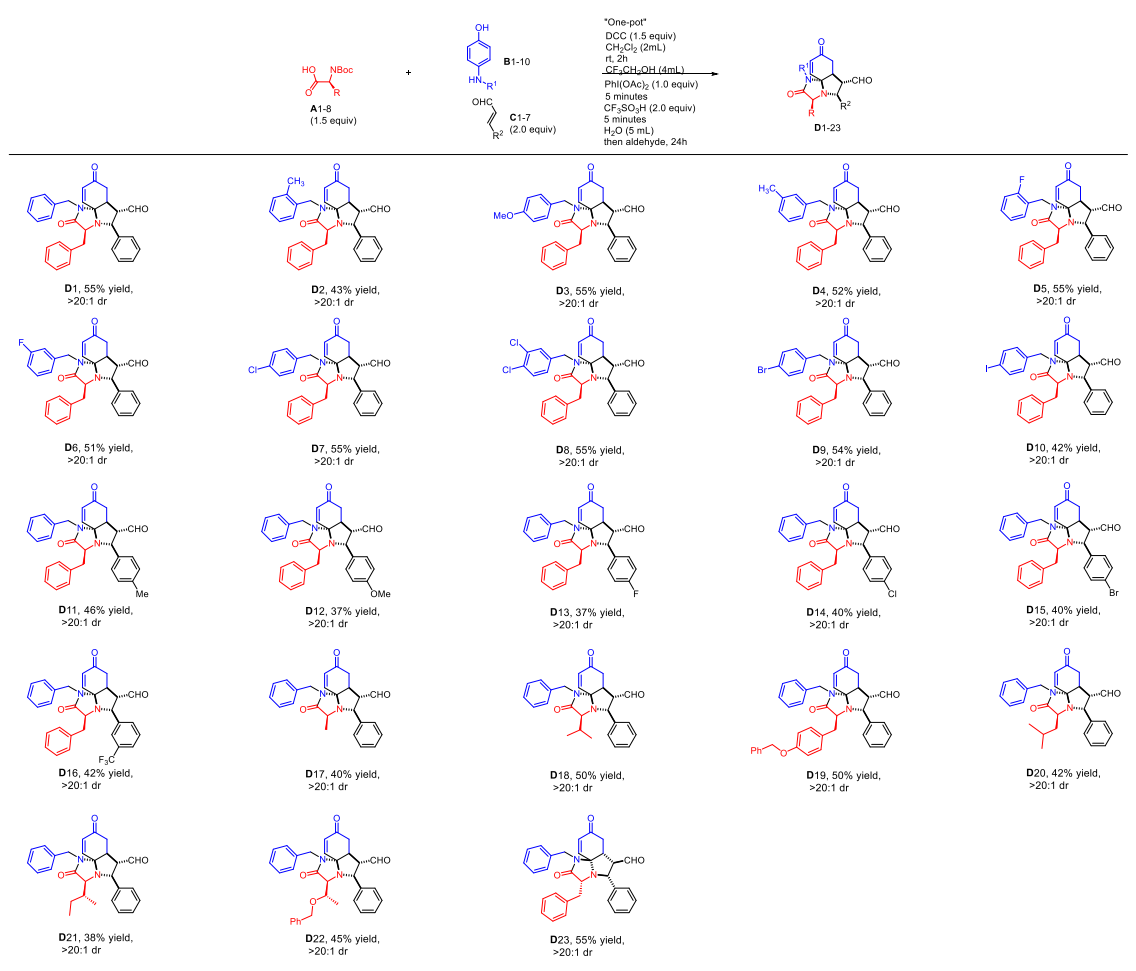


Scheme 5. Scale up synthesis of D1

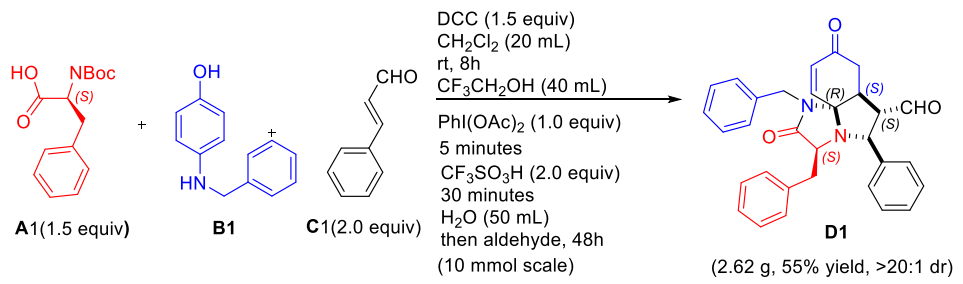

Table 1. Reaction screening for oxidative dearomatization reaction

\begin{tabular}{|c|c|c|c|c|}
\hline Entry & Oxidant & Solvent & Additive & Result \\
\hline 1 & $\mathrm{Phl}(\mathrm{OAC})_{2}$ & $\mathrm{Et}_{2} \mathrm{O}$ & None & No desired product \\
\hline 2 & $\mathrm{Phl}(\mathrm{OAc})_{2}$ & $\mathrm{CH}_{2} \mathrm{Cl}_{2}$ & None & No desired product \\
\hline 3 & $\mathrm{Phl}(\mathrm{OAC})_{2}$ & $\mathrm{CHCl}_{3}$ & None & No desired product \\
\hline 4 & $\mathrm{Phl}(\mathrm{OAC})_{2}$ & THF & None & No desired product \\
\hline 5 & $\mathrm{Phl}(\mathrm{OAc})_{2}$ & $\mathrm{C}_{6} \mathrm{H}_{5} \mathrm{CH}_{3}$ & None & No desired product \\
\hline 6 & $\mathrm{Phl}(\mathrm{OAC})_{2}$ & $\mathrm{CF}_{3} \mathrm{CH}_{2} \mathrm{OH}$ & None & $90 \%$ \\
\hline 7 & $\mathrm{Phl}(\mathrm{OAC})_{2}$ & $\mathrm{CH}_{3} \mathrm{OH}$ & None & No desired product \\
\hline 8 & $\mathrm{Phl}(\mathrm{OAc})_{2}$ & $\mathrm{CH}_{3} \mathrm{CN}$ & None & $35 \%$ \\
\hline 9 & $\mathrm{Phl}(\mathrm{OAC})_{2}$ & DMF & None & $25 \%$ \\
\hline 10 & IBX & $\mathrm{CF}_{3} \mathrm{CH}_{2} \mathrm{OH}$ & None & No desired product \\
\hline 11 & DMP & $\mathrm{CF}_{3} \mathrm{CH}_{2} \mathrm{OH}$ & None & No desired product \\
\hline 12 & $\mathrm{NaIO}_{4}$ & $\mathrm{CF}_{3} \mathrm{CH}_{2} \mathrm{OH}$ & None & No reaction \\
\hline 13 & $\mathrm{Phl}(\mathrm{OAC})_{2}$ & $\mathrm{CF}_{3} \mathrm{CH}_{2} \mathrm{OH}$ & Urea & $90 \%$ \\
\hline 14 & $\mathrm{Phl}(\mathrm{OAC})_{2}$ & TFE: $\mathrm{CH}_{3} \mathrm{CN}(3: 1)$ & Urea & $87 \%$ \\
\hline 15 & $\mathrm{Phl}(\mathrm{OAC})_{2}$ & TFE: $\mathrm{CH}_{3} \mathrm{CN}(2: 1)$ & Urea & $80 \%$ \\
\hline 16 & $\mathrm{Phl}(\mathrm{OAc})_{2}$ & TFE: $\mathrm{CH}_{3} \mathrm{CN}(1: 1)$ & Urea & $65 \%$ \\
\hline 17 & $\mathrm{Phl}(\mathrm{OAC})_{2}$ & TFE: $\mathrm{CH}_{2} \mathrm{Cl}_{2}(2: 1)$ & Urea & $85 \%$ \\
\hline
\end{tabular}


Table 2. Reaction screening for Aza-Michael/Michael reaction

\begin{tabular}{|c|c|c|c|}
\hline Entry & Acid HA (2 equiv) & Solvent & Result \\
\hline 1 & none & DCM & $0 \%$ \\
\hline 2 & TFA & Dichloromethane (DCM) & $90 \%$ \\
\hline 3 & PTSA & DCM & $0 \%$ \\
\hline 4 & Triflic acid & DCM & $0 \%$ \\
\hline 5 & $\mathrm{AcOH}$ & DCM & $35 \%$ \\
\hline 6 & $\mathrm{HCl} 37 \%$ & DCM & $0 \%$ \\
\hline 7 & $\mathrm{HBr} 49 \%$ & DCM & $0 \%$ \\
\hline 8 & $\mathrm{H}_{2} \mathrm{SO}_{4}$ & DCM & $0 \%$ \\
\hline 9 & $\mathrm{H}_{3} \mathrm{PO}_{4}$ & $\mathrm{DCM}$ & $0 \%$ \\
\hline 10 & TFA & DCM/TFE (1:2) & $0 \%$ \\
\hline
\end{tabular}

